\title{
The cosmic ray energy spectrum measured using the Pierre Auger Observatory
}

\author{
Francesco Fenu $^{* a b}$ for the Pierre Auger Collaboration ${ }^{c}$ \\ ${ }^{a}$ Università degli studi di Torino, Via Pietro Giuria 1, 10152 Torino, Italy \\ ${ }^{b}$ INFN Torino, Via Pietro Giuria 1, 10152 Torino, Italy \\ ${ }^{c}$ Observatorio Pierre Auger, Av. San Martín Norte 304, 5613 Malargüe, Argentina \\ E-mail: auger_spokespersons@fnal.gov \\ Full author list: http://www.auger.org/archive/authors_icrc_2017.html
}

\begin{abstract}
We present an update of the cosmic ray energy spectrum measured from $3 \times 10^{17} \mathrm{eV}$ to over $10^{20} \mathrm{eV}$ at the Pierre Auger Observatory. This energy range is covered by combining data from the surface detector (subdividing the events into a vertical and a horizontal data set), from a denser array of stations and from hybrid events simultaneously recorded by the surface and the fluorescence detectors. Data collected to date, with a total exposure exceeding $67,000 \mathrm{~km}^{2} \mathrm{sr}$ $\mathrm{yr}$, rely on the energy scale provided by the fluorescence detector. The spectral features, and the impact of systematic uncertainties on their significance, are discussed in detail.
\end{abstract}

35th International Cosmic Ray Conference - ICRC2017

10-20 July, 2017

Bexco, Busan, Korea

${ }^{*}$ Speaker. 


\section{Introduction}

The Pierre Auger Observatory [1] is the largest cosmic ray Observatory built so far. It is located in the Argentinian pampa near Malargüe, Mendoza province and has been in operation since 2004. The cosmic rays are studied by combining the measurements of a Surface Detector (SD) and a Fluorescence Detector (FD). The SD consists of 1600 Water Cherenkov Detectors (WCDs) on a $1500 \mathrm{~m}$ triangular grid (SD 1500), covering an area of $\sim 3000 \mathrm{~km}^{2}$, and of additional 61 detectors covering $23.5 \mathrm{~km}^{2}$ on a $750 \mathrm{~m}$ grid (SD 750). The SD 1500 is fully efficient at $3 \times 10^{18} \mathrm{eV}$ while the SD 750 from $3 \times 10^{17} \mathrm{eV}$ onwards. The atmosphere above the array is monitored by the 27 telescopes of the FD located in five buildings along the perimeter of the site. 24 telescopes have a field of view of $30^{\circ} \times 30^{\circ}$ in azimuth and elevation, with a minimum elevation of $1.51^{\circ}$ above the horizon. Three additional telescopes, the High Elevation Auger Telescopes, can cover an elevation up to $60^{\circ}$ to detect the low energy showers in coincidence with the SD 750. The FD measurements provide an almost calorimetric estimate of the shower energy but are limited by the atmospheric conditions while the SD measurements are made nearly $100 \%$ of the time. The main advantage of a hybrid system that combines the FD and SD measurements is the good control of the systematic uncertainties in the energy scale. It is possible calibrate the SD signal by exploiting the events where a simultaneous measurement of SD signal and FD energy is made, thus largely avoiding the use of Monte Carlo to reconstruct the energy.

In this contribution we present the energy spectrum measured at the Pierre Auger Observatory using an exposure exceeding 67,000 km² sr yr cumulated since January 2004 until December 2016. The measurements benefit from an improved reconstruction of the FD and SD events which will be described in detail.

\section{Improvements in the event reconstruction}

An accurate reconstruction of the FD events is complex, since it requires the knowledge of parameters like the fluorescence yield, the atmospheric conditions, the absolute calibration of the telescopes and many others. The entire procedure allows us to reconstruct the longitudinal profile of the energy deposit $(d E / d X)$ of the air shower in the atmosphere. Finally, from $E_{\text {cal }}=\int(d E / d X) d X$, which represents the energy deposited by the shower in atmosphere, the total energy is obtained by adding the so-called invisible energy, which is the energy carried into the ground by high energy muons and neutrinos. The analysis used to obtain the Auger energy scale and the estimate of its systematic uncertainties has been presented in [2]. Since this work, we have refined the reconstruction of the FD events obtaining an improved determination of the shower energies.

The reconstruction technique of the central laser facility of the Observatory, used to obtain the hourly measurements of the vertical aerosol optical depth, has improved, and now accounts for the shape of the aerosol scattering phase function and for the multiple scattering in the atmosphere. The two improvements cause an increase in the aerosol optical depth and consequently the shower energy increases of about $1 \%$ to $3 \%$ [3].

We have also improved the calibration of the FD telescopes. In the previous estimation, the optical efficiency, which is the relative FD response at various wavelengths, was the same for all telescopes. Now, after a dedicated campaign of measurements, we use the optical efficiency 
appropriate to each telescope which allows us to correctly take into account the different materials of which the mirrors are made [4]. We furthermore improved the estimation of the photomultiplier calibration constants used for the first years of data taking, during the construction phase of the Observatory. The shower energy is only marginally affected by all the improvements in the detector modelling with an average increase of $1 \%$.

Another improvement concerns the reconstruction of the longitudinal profiles of the showers. The measured $d E / d X$ is fitted using a Gaisser-Hillas (GH) function, but when only part of the energy deposited is detected, the extrapolation of the GH profile beyond the range of the measurements can introduce significant uncertainties in the determination of the electromagnetic shower energy. This happens in the showers of low energy $\left(<10^{18} \mathrm{eV}\right)$, for which only the flux of photons coming from the atmospheric depths around the profile maximum is bright enough to dominate over the night sky background. This problem has been solved by introducing a Gaussian constraint on the ratio $k=\frac{E_{\mathrm{cal}}}{(d E / d X)_{\max }}$ in the likelihood minimization procedure to fit the $d E / d X$ profile, where $(d E / d X)_{\max }$ is the energy deposited close to the maximum. The value of the constraint

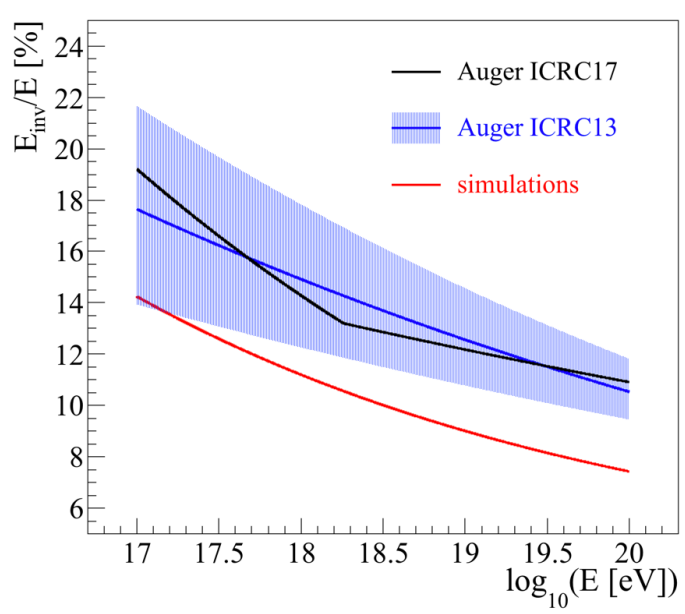

Figure 1: Ratio of the invisible energy to the total shower energy as a function of energy. The new Auger estimation (ICRC17) is compared with the previous parameterisation (ICRC13) [8] (the shaded band represents its systematic uncertainty) and with the one obtained by simulations. has been parameterised as a function of $E_{\mathrm{cal}}$ using an average of the QGSJetII-04 [5], EPOS-LHC [6], Sibyll2.3 [7] predictions with a mixed proton and iron composition and has been set to $k=\left(332.6+13.67 \log _{10} E_{\mathrm{cal}}\right) \mathrm{g} / \mathrm{cm}^{2}$. The uncertainty $\sigma_{k}$ is calculated as the standard deviation of $\mathrm{k}$, taking into account the different models used and the different composition simulated, and amounts to $29 \mathrm{~g} / \mathrm{cm}^{2}$. Both the parameter $\mathrm{k}$ and $\sigma_{k}$ are used in the likelihood function to constrain the GH fit. The new constraint improves the reconstruction of the low energy events, while its effect becomes negligible at energies $>10^{18}$ $\mathrm{eV}$. A more precise determination of the shower axis and a better pixel selection is also performed leading to a decrease in the shower energy by less than $1 \%$.

The estimation of the invisible energy $\left(E_{\text {inv }}\right)$ has been also improved. Our previous estimate was derived from events detected simultaneously by the FD and SD detectors (hybrid events) with zenith angles below $60^{\circ}$ [8]. In this work, $E_{\text {inv }}$ is estimated from the SD events with zenith angles between $60^{\circ}$ and $80^{\circ}$ and with energies above $4 \times 10^{18} \mathrm{eV}$ [9]. The advantage of using these showers is that the electromagnetic component is largely absorbed by the atmosphere and the signal in the SD detectors is dominated by muons. The estimator of the muon content in the shower $R_{\mu}$ [10] is well correlated with $E_{\text {inv }}$ through a power law function $E_{\text {inv }}=C R_{\mu}^{\delta}$. The coefficients $C$ and $\delta$ have been determined using the QGSJetII-04 interaction model with a mixed composition of protons and iron nuclei $\left(C=0.71 \times 10^{18} \mathrm{eV}\right.$ and $\left.\delta=0.96\right)$ and then used to estimate $E_{\text {inv }}$ from 
the measurements of $R_{\mu}$. In this way, most of the systematic uncertainties in $E_{\text {inv }}$ related to the predictions of the muon size are avoided. To extend the invisible energy evaluation to events below $60^{\circ}, E_{\text {inv }}$ is parameterised as a function of $E_{\text {cal }}$ based on an analysis of the hybrid events. This parameterisation is valid only above $4 \times 10^{18} \mathrm{eV}$ where the $R_{\mu}$ measurements are performed. The extrapolation to lower energies is obtained taking into account the evolution with energy of the mean mass composition measured at the Auger Observatory[11]. This is done using model-based functions with parameters that have been fixed to match the measurements at higher energies, finally obtaining:

$$
\begin{array}{ll}
E_{\text {inv }}=f_{\theta} a\left(\frac{E_{\mathrm{cal}}}{10^{18} \mathrm{eV}}\right)^{b} \times 10^{18} \mathrm{eV} & E_{\text {cal }}>E_{\text {cal }}^{A} \\
E_{\text {inv }}=f_{\theta} a\left(\frac{E_{\mathrm{cal}}^{A}}{10^{18} \mathrm{eV}}\right)^{b}\left(\frac{E_{\mathrm{cal}}}{E_{\mathrm{cal}}^{A}}\right)^{b_{\mathrm{extr}}} \times 10^{18} \mathrm{eV} & E_{\mathrm{cal}}<E_{\mathrm{cal}}^{A}
\end{array}
$$

where $a=0.1633, b=0.9463, b_{\text {extr }}=0.8475 . E_{\mathrm{cal}}^{A}=1.67 \times 10^{18} \mathrm{eV}$ is the energy at which the measurements made at the Observatory show a break in the elongation rate of the slant depth of the shower maximum $\left(X_{\max }\right)$ and $f_{\theta}=0.957$ is a factor that allows us to get an unbiased $E_{\text {inv }}$ estimation for the majority of the events with zenith angles $\theta<60^{\circ}$, given that the invisible energy tends to be larger for showers at larger zenith angles. The new $E_{\text {inv }}$ estimation is shown in Fig.1. It is fully consistent with our previous measurements, the only difference being the slope. The change arises from the improved sensitivity to the evolution of the mass composition with energy. The difference between our estimate and the one obtained using Monte Carlo (shown in Fig.1 by the red line) is a consequence of the well-known deficit of muons in the simulations, demonstrated in various analyses of the Auger data [12][13].

With the improved reconstruction presented in this paper, the cumulative energy shift of the FD energies is slightly energy dependent (larger at higher energies) and below 4\%. The total uncertainty of $14 \%$ in the energy scale and the uncertainties estimated for each sector of the reconstruction [2] are not significantly affected by the improvements in the FD reconstruction discussed above.

\section{The energy spectrum from SD 1500 events under 60 degrees}

The energy estimator of the SD events with zenith angles below $60^{\circ}$ is based on the lateral distribution of secondary particles on ground at an optimal distance from the shower core. For the SD 1500, the optimal distance is determined empirically and is $1000 \mathrm{~m}$. The seasonal and diurnal variations in the atmospheric parameters affect the distribution of the charged particles on ground and therefore the energy estimators. Such effects are corrected by modelling the dependence of the signal on the atmospheric parameters [14]. The presence of the geomagnetic field is accounted for [15].

Because of the attenuation of the shower when crossing the atmosphere, $S(1000)$ decreases with zenith angle for a given energy. Assuming an isotropic flux of cosmic rays, this dependence can be removed by using the Constant Intensity Cut method [16] converting S(1000) to the equivalent signal at median zenith angle of $38^{\circ}\left(S_{38}\right)$. 
The correlation between the SD energy estimator $S_{38}$ and the calorimetric energy $E_{\mathrm{FD}}$, measured by the FD, can be well described by a simple power law function $E_{F D}=A\left(S_{38}\right)^{B}$ [17]. The parameters $A$ and $B$ are obtained through a fit on a sub-sample of high quality hybrid events in the energy range of full efficiency of the SD.

The events are selected if the detector with the highest signal is enclosed in an hexagon of 6 active stations and the exposure is obtained geometrically [18]. A first estimate of the flux (the raw flux) is then obtained.

Finite energy resolution and the consequent bin migration, are accounted for using a forward folding procedure. The result is an energy dependent factor $C(E)$, which is the correction to be applied to the raw flux $J_{\text {unfolded }}=C(E) J_{\text {raw }}$. The correction to the flux by this procedure is of the order of $8 \%$ at $3 \times 10^{18} \mathrm{eV}, 1 \%$ at $10^{19}$ $\mathrm{eV}$ and $10 \%$ at $10^{20} \mathrm{eV}$.

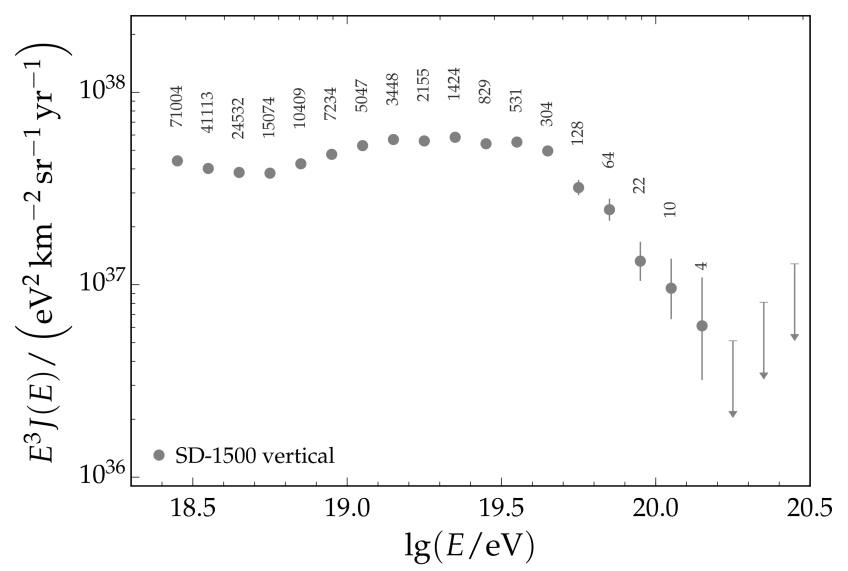

Figure 2: The unfolded spectrum for the SD 1500 vertical sample. The number of events is shown for each bin. The error bars represent statistical uncertainties. The upper limits correspond to the $84 \%$ C.L.

The SD 1500 vertical spectrum shown here includes data from January 2004 to December 2016, with a total exposure of 51,588 $\mathrm{km}^{2}$ sr yr (roughly 20\% higher than [19]). Several quality cuts are required: space-time coincidence of at least 3 neighboring triggering stations, containment of the events into an active hexagon and successful reconstruction of the lateral distribution of the events. Events detected in periods with problems in communication systems or in the vicinity of lightnings are excluded.

A total of 183,332 events with zenith angles below $60^{\circ}$ and energies above $3 \times 10^{18} \mathrm{eV}$ is selected. The spectrum is shown in Fig. 2 where we clearly see the ankle around $5 \times 10^{18} \mathrm{eV}$ and a steepening at the highest energies.

The events collected in the SD 1500 vertical spectrum cover a wide range of declinations from $-90^{\circ}$ to $25^{\circ}$ (more than $70 \%$ of the sky). This, along with the large cumulated exposure, can be exploited to investigate possible dependences of the spectrum on the declination. Data have been divided in two declination bands, $\left(-90^{\circ},-15.7^{\circ}\right)$ (south) and $\left(-15.7^{\circ}, 25^{\circ}\right)$ (north) and the spectrum has been evaluated for each sample. This choice also allows us to compare the spectrum measured by the Pierre Auger Observatory with the one by Telescope Array in the common declination band [20].

We show in Fig.3 (left) the spectrum divided in declination bands together with the overall one; the residuals with respect to the overall spectrum are plotted in the right panel. 

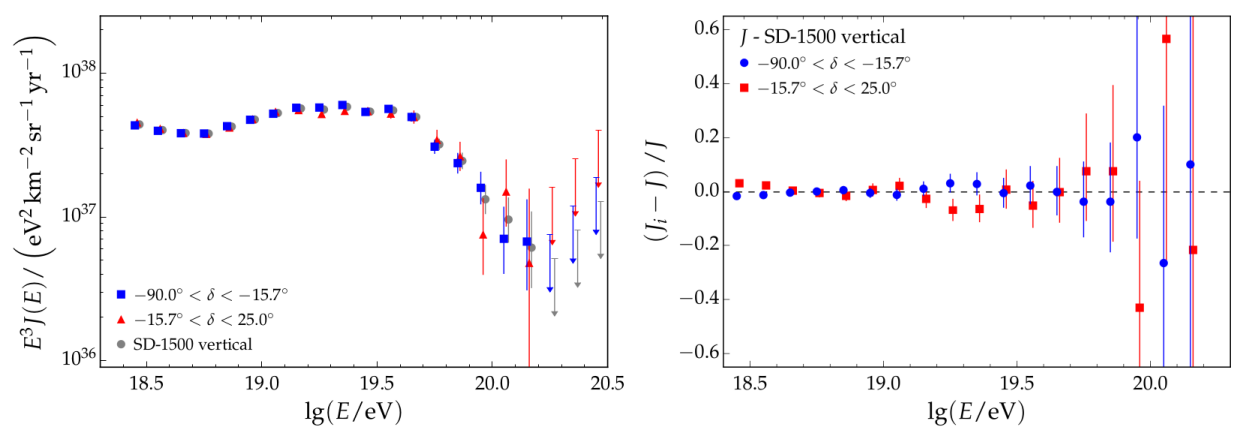

Figure 3: Left panel: The spectrum in declination bands (blue and red points), compared to the overall one (in gray). Right panel: the residual of the spectra in the various declination bands with respect to the overall spectrum.

\section{Other measurements of the energy spectrum}

Different data samples (see Tab.1 for their parameters) can be used from the Pierre Auger Observatory to derive the energy spectrum. Similar approaches are used to obtain each of the spectra but there are minor differences. The SD 750 energy estimator is the signal measured at $450 \mathrm{~m}$ from the core, $\mathrm{S}(450)$, corrected to a reference zenith angle of $35^{\circ}, \mathrm{S}_{35}$. The SD 1500 events with zenith angles above $60^{\circ}$ are reconstructed with an estimation of the relative muon content $N_{19}$ with respect to a simulated proton shower with energy $10^{19} \mathrm{eV}$ [10]. Finally, the hybrid sample is built from events detected by the FD simultaneously with at least one

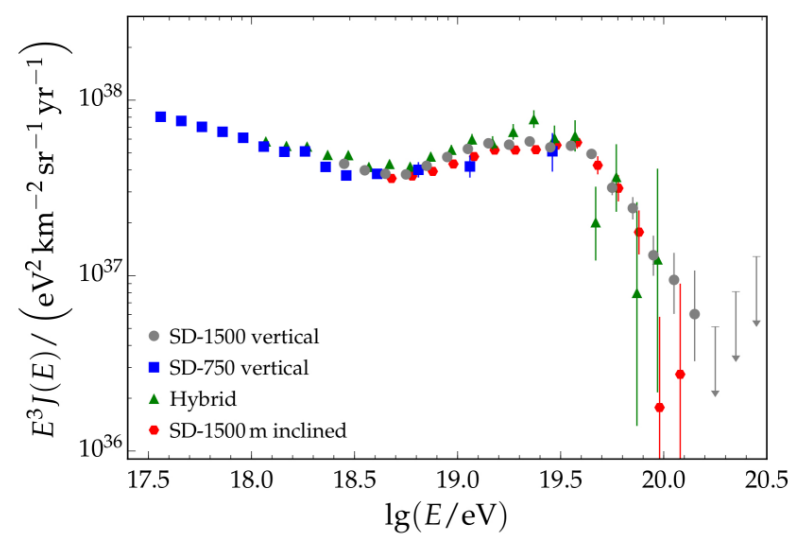

Figure 4: The energy spectra obtained with SD 1500 vertical, inclined, hybrid and SD 750 events are shown here. The systematic uncertainty on the energy scale, common to all of them, is $14 \%$ detector of the SD 1500. The hybrid exposure is calculated using a detailed Monte Carlo simulation [21].

The SD 1500 spectra obtained with events below and above $60^{\circ}$, the SD 750 and the hybrid spectra are shown together in Fig.4.

All the spectra agree within the systematic uncertainties, which are dominated by the energy scale one (14\%). The systematic uncertainties on the flux are between 5 and $10 \%$ and are responsible for the difference in normalization between the spectra visible in Fig.4.

A combined spectrum is obtained by means a maximum likelihood fit. The likelihood function is defined in such a way as to fit all the four data sets globally. The flux normalizations are used as additional constraints to obtain the flux scaling factors that match them: $(-0.8 \pm 0.2) \%$ for the SD 1500 vertical, $(-1 \pm 4) \%$ for the SD 750, $(5.4 \pm 0.7) \%$ for the SD 1500 horizontal and $(-6 \pm 2) \%$ for the hybrid. 


\begin{tabular}{|c|c|c|c|c|}
\hline & SD $1500<60^{\circ}$ & SD 1500 $>60^{\circ}$ & SD 750 & Hybrid \\
\hline Data taking period & Jan. 2004 - Dec. 2016 & Jan. 2004- Dec. 2016 & Aug. 2008 - Dec. 2016 & Jan. 2007 - Dec 2015 \\
\hline Exposure [km ${ }^{2}$ sr yr] & 51,588 & 15,121 & 228 & $1946 @ 10^{19} \mathrm{eV}$ \\
\hline Number of events & 183,332 & 19,602 & 87,402 & 11,680 \\
\hline Zenith angle range [deg.] & $0-60$ & $60-80$ & $0-55$ & $0-60$ \\
\hline Energy threshold [eV] & $3 \times 10^{18}$ & $4 \times 10^{18}$ & $3 \times 10^{17}$ & $10^{18}$ \\
\hline \multicolumn{5}{|c|}{ Calibration parameters } \\
\hline Number of events & 2661 & 312 & 1276 & \\
\hline A [eV] & $(1.78 \pm 0.03) \times 10^{17}$ & $(5.45 \pm 0.08) \times 10^{18}$ & $(1.4 \pm 0.04) \times 10^{16}$ & $1.000 \pm 0.008$ \\
\hline B & $1.042 \pm 0.005$ & $1.030 \pm 0.018$ & 13 & \\
\hline Energy resolution [\%] & 15 & 17 &
\end{tabular}

Table 1: The parameters of the data samples presented here together with the calibration parameters.

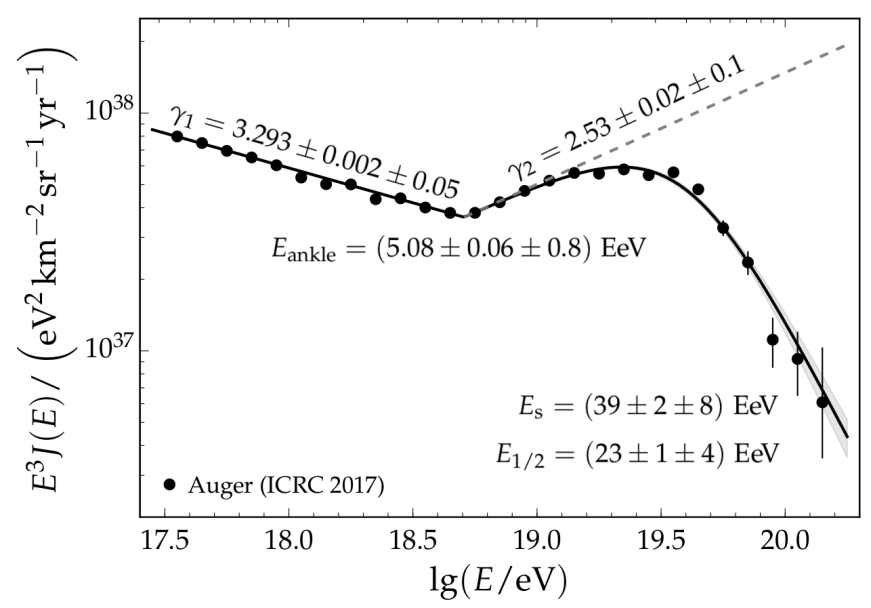

Figure 5: The combined spectrum and the fitting function with the fitting parameters.

To obtain the spectral parameters, the combined spectrum is fitted with the function:

$$
J_{\text {unf }}(E)= \begin{cases}J_{0}\left(\frac{E}{E_{\text {ankle }}}\right)^{-\gamma_{1}} & E<E_{\text {ankle }} \\ J_{0}\left(\frac{E}{E_{\text {ankle }}}\right)^{-\gamma_{2}}\left[1+\left(\frac{E_{\text {ankle }}}{E_{\mathrm{s}}}\right)^{\Delta \gamma}\right]\left[1+\left(\frac{E}{E_{\mathrm{s}}}\right)^{\Delta \gamma}\right]^{-1} & E>E_{\text {ankle }}\end{cases}
$$

The spectrum, the fit and the optimized parameters are plotted in Fig.5. An ankle is found at $E_{\text {ankle }}=(5.08 \pm 0.06$ (stat. $) \pm 0.8$ (syst. $\left.)\right) \times 10^{18} \mathrm{eV}$, while the suppression is at $E_{s}=(3.9 \pm 0.2$ (stat. $) \pm$ 0.8 (syst. $)) \times 10^{19} \mathrm{eV}$. The energy $\mathrm{E}_{1 / 2}$ at which the integral spectrum drops by a factor of two below what would be the expected with no steepening is $\mathrm{E}_{1 / 2}=(2.26 \pm 0.08$ (stat.) \pm 0.4 (syst.) $) \times 10^{19}$ $\mathrm{eV}$. The spectral indexes are: $\gamma_{1}=3.293 \pm 0.002$ (stat.) \pm 0.05 (syst.), $\gamma_{2}=2.53 \pm 0.02$ (stat.) \pm 0.1 (syst.) while $\Delta \gamma=2.5 \pm 0.1$ (stat.) \pm 0.4 (syst.).

\section{Summary}

We have presented an update of the energy spectrum above $3 \times 10^{17} \mathrm{eV}$ as obtained using the Pierre Auger Observatory. An improved FD reconstruction caused an increase in the FD energy of less than $4 \%$, while the systematic uncertainties previously estimated by the Auger Collaboration 
are confirmed. The SD 1500 vertical spectrum has been obtained with unprecedented precision based on an exposure of more than $51,000 \mathrm{~km}^{2} \mathrm{sr}$ yr. A study of the declination dependence of the spectrum showed no significant north-south asymmetry.

The combined spectrum from four different data sets has been derived using data collected by the Pierre Auger Observatory over more than 10 years (cumulating $67,000 \mathrm{~km}^{2} \mathrm{sr} \mathrm{yr}$ of exposure). The measured cosmic ray flux is well described by a broken power law plus a smooth suppression at the highest energies. The dominant systematic uncertainty stems from the overall uncertainty in the energy scale of $14 \%$. The obtained spectral parameters are in good agreement with those previously shown [19].

\section{References}

[1] The Pierre Auger Collaboration, Nucl. Instrum. Meth. A 798 (2015) 172.

[2] V. Verzi for the Pierre Auger Collaboration, Proc. 33th ICRC 2013, Rio de Janeiro, Brazil [arXiv:1307.5059].

[3] M. Malacari for the Pierre Auger Collaboration, Proc 35th ICRC 2017, this conference.

[4] B. Gookin, doctoral thesis, https://dspace.library.colostate.edu/bitstream/handle/10217/167192/ Gookin_colostate_0053A_13168o.pdf?sequence=4\&isAllowed=y

[5] S. Ostapchenko, Phys. Rev. D 83 (2011) 014018.

[6] T. Pierog, Phys. Rev. C 92 (2015) 034906.

[7] F. Riehn, Proc. 34th ICRC 2015, The Hague, The Netherlands [arXiv:1510.00568].

[8] M. Tueros for the Pierre Auger Collaboration, Proc. 33th ICRC 2013, Rio de Janeiro, Brazil [arXiv:1307.5059]

[9] A. Mariazzi for the Pierre Auger Collaboration, Proc. of the 2016 Conference on Ultrahigh Energy Cosmic Rays, Kyoto, Japan (2016), in press.

[10] The Pierre Auger Collaboration, JCAP 08 (2014) 019.

[11] A. Porcelli for the Pierre Auger Collaboration, Proc. 34th ICRC 2015, The Hague, The Netherlands. PoS(ICRC2015)420.

[12] The Pierre Auger Collaboration, Phys. Rev. D 91, 032003 (2015)

[13] The Pierre Auger Collaboration, Phys. Rev. Lett. 117 (2016) 192001.

[14] The Pierre Auger Collaboration, JINST 12 (2017) P02006.

[15] The Pierre Auger Collaboration, JCAP11 (2011) 022.

[16] J. Hersil et al., Phys. Rev. Lett. 6 (1961) 22.

[17] R. Pesce for the Pierre Auger Collaboration, Proc. 32nd ICRC 2011, Beijing, China, DOI: 10.7529/ICRC2011/V02/1160.

[18] The Pierre Auger Collaboration, Nucl. Instrum. Meth. A 613 (2010) 29.

[19] I. Valiño for the Pierre Auger Collaboration, Proc. 34th ICRC 2015, The Hague, The Netherlands. PoS(ICRC2015)271.

[20] D. Ivanov for the Pierre Auger and Telescope Array collaborations, Proc. 35th ICRC 2017, this conference

[21] The Pierre Auger Collaboration, Astropart. Phys. 34 (2011) 368-381. 